\title{
Entre connaissance et croyance : Kang Youwei et le destin moderne du confucianisme
}

Between Knowledge and Faith: Kang Youwei and the Modern Fate of

Confucianism

知識與信仰之間: 康有為與儒學的現代命運

\section{Chunsong Gan}

Traducteur : Aurore Merle et Guillaume Dutournier

\section{(2) OpenEdition}

\section{Journals}

Édition électronique

URL : http://journals.openedition.org/extremeorient/189

DOI : 10.4000/extremeorient.189

ISSN : 2108-7105

Éditeur

Presses universitaires de Vincennes

Édition imprimée

Date de publication : 1 novembre 2011

Pagination : 115-142

ISBN : 978-2-84292-334-1

ISSN : 0754-5010

\section{Référence électronique}

Chunsong Gan, « Entre connaissance et croyance : Kang Youwei et le destin moderne du

confucianisme », Extrême-Orient Extrême-Occident [En ligne], 33 | 2011, mis en ligne le 01 novembre

2014, consulté le 21 avril 2019. URL : http://journals.openedition.org/extremeorient/189; DOI :

10.4000/extremeorient.189 


\title{
Entre connaissance et croyance: Kang Youwei et le destin moderne du confucianisme
}

\author{
Gan Chunsong
}

\section{Introduction : le confucianisme est-il un système de savoir ou de croyance?}

En 1893, lors du «Parlement des religions du monde» à Chicago, Peng Guangyu, premier secrétaire de la délégation chinoise aux États-Unis, s'efforça d'expliquer la différence entre le «jiao» du mot «rujiao» (confucianisme) et la notion occidentale de «religion». Il déclara notamment: «En Chine, le jiao (l'instruction) équivaut au zheng (le politique) et le zheng équivaut au jiao. Le zheng et le jiao sont tous deux issus du Fils du Ciel. L'instruction dispensée par l'empereur (dijiao) et celle délivrée par les maîtres (shijiao) sont toutes deux une instruction portant sur les rites (lijiao). En dehors de cette instruction, aucune église ne commande au peuple en Chine ${ }^{1}$. Peng considérait que le caractère $w u$ (dans le sens de magie, de chamanisme) était préférable au mot jiao comme équivalent du terme anglais «religion». Il s'était rendu au constat que le confucianisme, source de l'éducation et instance de transformation du peuple chinois, ne pouvait être compris comme une «religion». Un constat largement partagé parmi les élites intellectuelles de l'époque: même Kang Youwei (1858-1927), l'un des leaders du mouvement de réforme des Cent jours (11 juin - 21 septembre, 1898) qui pourtant s'efforça de transformer le confucianisme en un système de croyances religieuses, jugeait cette traduction inadéquate. Pour ce dernier, en traduisant «religion» par zongjiao, les Japonais désignaient principalement «l'enseignement divin» (shenjiao), ce que Peng Guangyu qualifiait précisément de $w u$ (chamanisme):

1. Peng Guangyu, Shuo jiao, tome 1, p. 3. Concernant les débats sur la pénétration et les modifications du mot zongjiao dans la Chine moderne, voir: Chen Xiyuan, «"Zongjiao": yige Zhongguo jindai wenhuashi shang de guanjianci». 


\section{Gan Chunsong}

Le sens de «religion» ne se limite pas à l'enseignement divin. Seulement, influencé par le christianisme, on tend à croire qu'il n'y aurait pas de religion sans dieu. La combinaison de jiao et zong est déjà inappropriée pour exprimer le sens de «religion». Considérer que le confucianisme ne peut être une religion sous prétexte que, contrairement au bouddhisme, à l'islam et au christianisme qui professent tous une voie divine, Confucius n'abordait pas cette question, cela revient à connaître deux fois cinq tout en ignorant $\operatorname{dix}^{2}$.

Or, Kang Youwei considérait que zongjiao devait inclure la voie divine et la voie humaine. D'après lui, «dans la haute antiquité, les hommes incultes vouaient un culte aux esprits, si bien que l'enseignement divin était vénéré; avec la civilisation moderne, l'accent fut mis sur l'humain et la voie humaine gagna en importance. De fait, la voie humaine a suivi l'enseignement divin avant de le dépasser $»^{3}$.

Ce que Kang Youwei s'efforçait de souligner, c'était la différence entre le phénomène du jiaohua propre au confucianisme, c'est-à-dire la transformation du corps social par l'enseignement, et le shenjiao, c'est-à-dire l'enseignement divin. Il cherchait par là à faire ressortir la rationalité du confucianisme. Pourtant, il est faux de dire que le jiaohua n'avait aucun lien avec le shenjiao, la tradition confucéenne s'étant efforcée de combiner en un système composite la voie des hommes et celle des dieux - ou, pour le formuler autrement, d'intégrer croyances et connaissances.

Un trait essentiel du développement de la modernité européenne fut la séparation du politique, du savoir et de la foi, la religion ne contrôlant plus la totalité de l'existence. Comment répondre au défi de la modernité occidentale, et trouver un équilibre entre la nécessité d'apprendre de l'Occident et le souci de préserver l'identité de la nation? Telles étaient les questions centrales pour Kang Youwei. Pour parvenir à ses fins, il orienta son effort dans trois directions : premièrement, souligner la «rationalité» d'un confucianisme ne traitant «jamais de l'étrange ni des esprits, de la force brute ni des actes contre nature ${ }^{4}$, mais aussi réformer le système d'enseignement et notamment les examens mandarinaux (keju), afin de répondre aux besoins scientifiques et de permettre la diffusion des connaissances occidentales; deuxièmement, par la création d'une église, ériger Confucius en fondateur d'une religion à la signification complexe; troisièmement, redéfinir le rôle du confucianisme dans la construction de l'identité de la nation et dans l'éducation morale de la population.

2. Kang Youwei, «Kongjiaohui xu», p. 346.

3. Ibid.

4. Citation du Lипуи (Entretiens de Confucius), VII, 21 (traduction Anne Cheng, Seuil, 1981, p. 65). 
Ces trois missions entrant parfois en conflit, Kang Youwei n'obtint le soutien ni des conservateurs confucéens, ni des nouvelles élites intellectuelles formées dans des institutions modernes ou à l'étranger. Cet article, en se focalisant sur deux aspects mis en avant par Kang, la transformation du confucianisme en religion et la réforme du système traditionnel d'enseignement, examine les tentatives d'un certain confucianisme pour surmonter ses difficultés et s'adapter à une société moderne.

\section{La tentative de Kang Youwei de transformer le confucianisme en religion et son échec}

Liang Qichao (1644-1911), réformiste et disciple de Kang Youwei, eut un jour ce jugement selon lequel les gens se souviendraient de Kang Youwei comme d'un maitre religieux. Ce dernier savait parfaitement que dans le système religieux chinois, c'étaient le bouddhisme, le taoïsme ainsi que les nombreux cultes populaires qui constituaient le cœur de la culture religieuse. Kang Youwei était pourtant pleinement conscient que la séparation entre pouvoir politique et confucianisme ébranlerait la légitimité de ce dernier. C'est pourquoi il souhaitait, en réformant le confucianisme, c'est-à-dire en l'institutionnalisant comme une religion, lui forger un nouveau mode d'existence au sein de la société chinoise. Comme l'affirma Liang Qichao, Kang Youwei s'efforça de devenir le «Martin Luther» du confucianisme. Mais là où Martin Luther connut un immense succès, l'échec de Kang fut total.

\section{La conception d'une religion confucéenne avant la fondation de la République chinoise}

À ses débuts, Kang Youwei employait le mot jiao dans le sens de jiaohua (transformation par l'éducation). Il considérait que c'était à travers son rôle éducatif que le confucianisme fondait l'ordre social (ou la «voie humaine», rendao). Dans «Jiaoxue tongyi», écrit en 1885, il affirme:

L'établissement de l'éthique par l'enseignement des rites, ainsi que la maîtrise des savoir-faire concernant les affaires et les êtres, constituent la base sur laquelle se fonde la voie humaine. L'éthique de l'enseignement des rites s'adresse à la morale; les savoir-faire concernant les affaires et les êtres s'adressent à l'art. Ce que «le Saint de l'époque ultérieure» (housheng) ${ }^{5}$ dit de l'enseignement concerne ces deux éléments; ce qui est dit de l'apprentissage concerne ces deux éléments ${ }^{6}$.

5. «Housheng» est un titre parfois attribué à Xun $\mathrm{Zi}$, penseur confucéen au III ${ }^{\mathrm{e}}$ siècle av. J.-C.

6. Kang Youwei, «Jiaoxue tongyi», p. 20. 


\section{Gan Chunsong}

Or, au fur et à mesure que sa compréhension des croyances et de la science occidentales augmentait, sa conception du jiao devint à la fois plus étendue et plus concrète. Si l'étude des Classiques constitue traditionnellement le fondement de ce qu'on appelle jiaohua, Kang tenta d'y intégrer les savoirs occidentaux, mais aussi les «principes subtils découverts par les gens des cinq continents». Dans «Shili gongfa quanshu», rédigé en 1888, il propose une explication du terme jiao:

Il y a deux principes qui fondent l'éducation. Premièrement, augmenter l'intelligence et la compétence de l'éduqué, et renforcer ainsi sa capacité d'aimer et sa confiance en soi. Deuxièmement, lui enseigner les principes subtils et les excellentes institutions découverts par les gens des cinq continents, de sorte qu'il puisse en bénéficier pour vaincre ses travers et se débarrasser des malhonnêtetés auxquelles il peut être habitué. De cette façon, son intelligence et sa compétence ne risquent pas d'être dévoyés ${ }^{7}$.

Cette définition du jiaohua ajoutait donc à un objectif moral relativement classique un programme d'acquisition de connaissances scientifiques. À ce stade, il s'agissait encore de partir du jiaohua, autrement dit de «se fonder sur la Chine pour comprendre l'Occident ». Mais après 1890, Kang s'orienta davantage vers une restructuration du confucianisme à partir des modèles religieux occidentaux. Cela revenait à «se fonder sur l'Occident pour comprendre la Chine», mais ses détracteurs estimaient qu'il «transformait la Chine avec l'Occident».

Kang Youwei s'intéressait grandement au prosélytisme du christianisme, du bouddhisme et des autres religions. En 1891, dans une série de discussions avec Zhu Yixin (1846-1894), il présenta sa vision du prosélytisme, expliquant que l'acceptation de la grande voie de Confucius dépendait de deux choses: premièrement, développer et glorifier, deuxièmement, propager et diffuser. Pour lui, la raison principale de la pénétration du bouddhisme en Chine et de la non-diffusion du confucianisme en Inde n'était pas dans l'infériorité du confucianisme mais dans leur différence en matière de prosélytisme. Si, en dehors de l'Asie où régnaient encore le confucianisme, le bouddhisme et l'islam, la majorité des pays du monde avait foi dans le christianisme, c'était parce que l'Europe «excellait dans les arts de fabrication, de sorte que ses bateaux et véhicules pouvaient galoper hors de ses frontières $»^{8}$.

Après la guerre sino-japonaise en 1894, Kang Youwei commença à évoquer de manière systématique son projet de réforme. Dans les mémoires au trône qu'il rédigea pour d'autres personnes, il exposa à l'empereur Guangxu (règne

7. Kang Youwei, «Shili gongfa quanshu», p. 156.

8. Kang Youwei, «Yu Zhu Yixin lun xue shu du», p. 325. 
1875-1908) ses propositions pour remédier à l'arriération de la Chine. Un point critique de son projet était la préservation de la place de la pensée confucéenne.

Dans ses «Seconde » et «Troisième requête soumise à l'empereur» (Shang qingdi di er/san shu), Kang pose clairement la question de la construction d'une «discipline d'étude de la voie» (daoxueke), sa principale fonction consistant, à travers la diffusion du «dogme» (jiaoyi) de Confucius, à s'opposer au déclin croissant de la morale des Chinois. Dans sa requête, Kang avance même le projet de porter la bonne parole confucéenne à l'étranger.

À la veille de la réforme des Cent jours, le projet de Kang Youwei se précise. En 1898 (le $5^{\mathrm{e}}$ mois de la $24^{\mathrm{e}}$ année de Guangxu), dans une requête pour renouveler le confucianisme, il expose deux idées centrales:

Premièrement, il souhaite fonder une Église de la religion confucéenne (kongjiaohui) et régler ainsi une question délicate pour la Cour impériale concernant les affaires religieuses. Il propose également de faire des descendants directs de Confucius qui portent le titre héréditaire de «Ducs pour la multiplication des sages» (Yansheng Gong) l'équivalent des évêques dans la religion chrétienne.

Deuxièmement, Kang attribue la cause du déclin national au manque de talents, la pénurie de lettrés résidant dans le mode de sélection propre au système des examens impériaux, en particulier l'essai en huit parties (bagu) qui conduit les candidats à ne pas prêter attention aux Classiques confucéens. Pour restaurer la tradition confucéenne, il est donc indispensable de revenir aux textes originels. Selon la démonstration de Kang, certains Classiques, notamment les Entretiens, sont le fait des disciples de Confucius: seuls les Annales des Printemps et Automnes représentent la pensée éternelle du Maître.

Ici, le projet de réforme de Kang Youwei devient de plus en plus clair: il vise à séparer les affaires religieuses de la diffusion des connaissances. Kang estime que pour assurer la prospérité et la puissance du pays et s'adapter à la compétition internationale, il faut d'abord construire un système de croyances, puis réformer le système éducatif - ces deux objectifs étant indissociables. Fonder un système de croyances répond au souci de construire l'identité de la nation, tandis que la réforme éducative vise à former des talents et à augmenter le niveau général de la population, le but ultime étant de préserver la nation, la race et l'enseignement confucéen.

Cette nouvelle compréhension du confucianisme promue par Kang Youwei suscita de nombreuses critiques. Les premières provenaient du cœur du système confucianiste, notamment du «courant des textes anciens » (guwen jingxue) qui ne pouvait tolérer certaines expressions de Kang issues des «textes modernes» (jinwen jingxue), telles que «réforme des institutions» (gaizhi) ou «fondateur 
religieux» $(\text { jiaozhu })^{9}$. Dans un autre texte fondateur, «Xinxue weijing kao», Kang estimait qu'une part importante des écrits confucéens était de faux Classiques, ce qui provoqua l'ire de nombreux lettrés.

Concernant son projet de religion confucéenne (kongjiao), qu'il portait avec d'autres, certains critiques y voyaient l'importation camouflée d'une religion étrangère. Certains fonctionnaires impériaux affirmaient même que Kang Youwei souhaitait s'ériger en pape et partager le pouvoir politique avec l'empereur. L'un des effets négatifs de ces attaques fut que des personnages politiques majeurs comme Zhang Zhidong (1837-1909) retirèrent leur soutien politique et financier à Kang.

Même avec Liang Qichao, le disciple qui lui donnait le plus de fierté, des divergences existaient concernant ce projet de fondation religieuse. C'est pourquoi, après la fondation de la République en 1912, Kang confia la charge de ce projet à Chen Huanzhang (1880-1933) ${ }^{10}$. Quant à Liang, au tournant du $\mathrm{XIX}^{\mathrm{e}}-\mathrm{XX}^{\mathrm{e}}$ siècle, sous l'influence de Yan Fu (1854-1921) et de Huang Zunxian (1848-1905), il commença à mettre en doute la convergence entre la fondation d'une religion confucéenne et la réforme politique. Il doutait également que l'instauration d'une Église confucéenne pût exercer une influence positive sur la construction morale de la société. La divergence s'accrut après 1902 : «Après 1902, Qichao ne parla plus de "faux Classiques", ni de "réformer les institutions", tandis que son maître Kang Youwei promouvait vigoureusement l'idée de fonder une Église de la religion confucéenne, de l'ériger en religion d'État et de vénérer le Ciel et Confucius. Une vision qui ne manqua pas d'appui dans le pays. Qichao n'était pas d'accord et les réfuta à plusieurs reprises ${ }^{11}$. Dans un long article, «Baojiao fei suoyi zun Kong lun» (En défendant la religion confucéenne, on ne respecte pas les théories de Confucius), Liang insiste sur l'opposition entre ses positions passées et présentes, procédant ainsi à une analyse d'ensemble de son positionnement:

9. Après une longue période d'érudition historiciste et rationaliste, portant surtout sur les Classiques dits en «écritures ancienne» qui avaient été consacrés sous les Tang, la fin du $\mathrm{XVIII}^{\mathrm{e}}$ voit réapparaître une pratique exégétique visant des vérités atemporelles, qu'elle découvre essentiellement dans les Annales des Printemps et Automnes. Dans ce Classique, Confucius était censé avoir exprimé à mots couverts une «critique éthicopolitique qui visait ses contemporains autant qu'il dessinait un monde idéal à venir » (cf. Anne Cheng, Histoire de la pensée chinoise, p. 610). C'est dans ce dernier courant que s'inscrit Kang Youwei.

10. Chen Huanzhang, natif de Gaoyao dans le Guangdong et disciple de Kang Youwei, est un des représentants principaux du mouvement de fondation d'une religion confucéenne.

11. Ding Wenjiang \& Zhao Fengtian (éds.), Liang Qichao nianpu changbian, p. 279. 
Ceux qui promeuvent l'idée de protéger la religion confucéenne ignorent plusieurs choses. Premièrement, ils ne connaissent pas la vérité de Confucius; deuxièmement, ils ne comprennent pas la définition d'une religion ; troisièmement, ils ne voient pas les tendances futures des religions; quatrièmement, ils ignorent les rapports entre politique et religion dans les autres pays ${ }^{12}$.

Liang estimait que Confucius était un philosophe, un éducateur, un homme politique et non un fondateur de religion comparable aux figures occidentales. Par conséquent, la préservation de la religion confucéenne était un mouvement sans raison d'être. Parmi les personnages historiques chinois, seuls Zhang Daoling et d'autres figures fondatrices du taoïsme pouvaient être perçus comme des acteurs religieux. S'appuyant sur la théorie de l'évolutionnisme social en vogue à l'époque, Liang Qichao considérait la religion comme une culture déclinante. Suivre le modèle des religions occidentales était donc à ses yeux une attitude d'imitation aveugle.

\section{Le conflit entre religion d'État et liberté de croyance après la fondation de la République de Chine}

En 1905, à la suite de l'abolition des examens impériaux, le confucianisme perd son soutien institutionnel le plus important, tandis qu'avec le déclenchement de la révolution de 1911 et la fondation de la République de Chine, la Chine devient la première république d'Asie. Dans ce nouveau régime, le confucianisme perd sa «suprématie», ses Classiques n'étant plus la source de la vie politique et sociale. La création de nouvelles écoles suivant le modèle occidental fait également perdre au confucianisme le soutien dont il disposait dans le domaine de l'éducation.

Dans ces nouvelles écoles, la question qui faisait débat depuis 1901 - le contenu principal de l'éducation devait-il être le savoir occidental ou le savoir chinois? - fut définitivement tranchée: les Classiques confucéens, autrefois fondement des valeurs chinoises, furent séparés puis intégrés au système scientifique moderne, devenant des «savoirs» parmi d'autres et non plus la «voie» qui commandait au savoir.

Kang Youwei, en exil prolongé après l'échec de la réforme des Cent jours, en ressentait une vive inquiétude. Dans le chaos politique qui suivit la fondation de la République, il identifia la crise politique à une crise de valeur. La solution à cette crise était de revenir au confucianisme et d'ériger la religion confucéenne en religion d'État.

12. Liang Qichao, «Baojiao fei suoyi zun Kong lun», p. 307. 
Durant les premières années de la République, il affirme dans une lettre adressée à Chen Huanzhang (le 30 juillet 1912) :

Aujourd'hui, il est bien difficile de faire fonctionner un parti politique. Les différents partis se nuisent réciproquement [...]. Auparavant, lorsque, aux États-Unis, vous avez pris la responsabilité de promouvoir la religion confucéenne, votre recherche et vos prises de paroles se faisaient en toute clarté. Désormais, si vous vous présentiez comme un missionnaire et lanciez le débat sur l'abandon du confucianisme, il y aurait certainement des échos. Cela n'inquiéterait pas les partis politiques et serait donc plus facile à réaliser ${ }^{13}$.

Comme nous le montre cette lettre, l'instauration d'une religion confucéenne ne vise pas simplement à fonder une religion, mais bien, grâce à la puissance d'action offerte par le modèle de l'église, à déployer son influence sur la politique. Dans le contexte politique des premières années de la République, Kang considérait que créer un parti politique n'aurait pas autant d'efficacité que fonder une Église de la religion confucéenne.

En dehors du fait que celle-ci comportait moins de risques qu'un parti, il y avait là la possibilité de s'appuyer sur le groupe des lettrés traditionnels marginalisés par la nouvelle société. «Aujourd'hui, les lettrés et étudiants issus de l'ancien système sont encore partout dans le pays. Si on les invite à rejoindre cette Église, ils s'y montreront certainement favorables ». Cependant, Kang avait conscience que la proposition d'un nouveau rapport de réciprocité entre souverain et sujets serait en porte-à-faux avec la nouvelle situation politique. La promotion de la religion confucéenne devait connaître des modifications : «J'ai annoté le Liyun, le Zhongyong, les Sishu, le Chunqiu ainsi que des extraits du Liji. On pourra les diffuser pour répandre mes idées de "paix" (shengping), de "grande paix" (taiping) et de "grande unité" (datong), de sorte que les gens ne doutent plus de la possibilité du développement du confucianisme après l'abolition de la relation du monarque et des sujets ${ }^{14}{ }$. Très confiant dans l'avenir, Kang Youwei pensait qu'en commençant dans chaque province, en à peine six mois, les églises de la religion confucéenne se répandraient dans chaque préfecture et district.

En septembre et octobre 1912, dans deux avant-propos sur l'Église de la religion confucéenne («Kongjiaohui xu»), il commence à élaborer sa théorie. La perspective y est de nouveau celle du jiaohua, c'est-à-dire de la transformation du corps social par l'éducation. Pour Kang, la société chinoise enviait l'Europe et les États-Unis, mais ignorait que leur développement reposait sur la coexistence entre le politique, les conditions matérielles et le jiaohua - politique et jiaohua

13. Kang Youwei, «Yu Chen Huanzhang shu», p. 337.

14. Ibid. 
étant mutuellement dépendants. L'exposé de Kang Youwei sur le sens et le contenu de cette religion s'appuie en réalité sur les éléments suivants:

Premièrement, concernant les relations entre religion et identité nationale. Comme l'explique Kang, dans le cas des Juifs, l'État a été détruit mais l'enseignement (religieux) n'ayant pas disparu, le pays peut par conséquent renaître. À l'inverse, au Mexique, l'annexion culturelle a conduit les Mexicains à se considérer comme les descendants des seuls Espagnols. Par conséquent, la décision du ministère de l'Éducation chinois de supprimer l'enseignement de Confucius fera perdre à la Chine le fondement de son identité nationale.

Deuxièmement, le développement de nombreuses religions dans le monde étant lié aux activités de prosélytisme, la religion confucéenne devrait également encourager de telles activités.

Troisièmement, confronté à l'argument selon lequel la religion confucéenne serait en contradiction avec un régime républicain égalitaire, Kang Youwei concède que la relation entre le maître et ses sujets est verticale, mais il rappelle que la religion confucéenne affirme que toute chose, sous le ciel, est un bien public. Par conséquent, cette religion se trouve en conformité, et même en avance, avec les principes politiques en vigueur.

Quatrièmement, analysant les relations entre «religion» et jiao, Kang affirme qu'indépendamment de la croyance en une divinité, tout système de croyances peut s'appeler «religion» (zongjiao). Les Japonais ayant utilisé les caractères chinois «zongjiao» pour traduire le terme de religion, celle-ci a été comprise comme un théisme. Si certains Chinois contestent à Confucius le statut de fondateur d'une religion sous prétexte qu'il n'a pas parlé de Dieu, c'est essentiellement parce qu'ils ont subi l'influence de la langue japonaise qui fait un usage erroné des caractères chinois ${ }^{15}$.

Comme nous le montre le projet de religion confucéenne, la compréhension que Kang Youwei a du «jiao» oscille entre l'enseignement traditionnel et la religion, morale et religion étant ici assimilées. Il souhaite à la fois que le confucianisme conserve une haute position spirituelle, tout en réduisant la « distance» séparant le confucianisme de la politique républicaine et de la science moderne. Pour Kang, fonder une religion est la voie la plus sûre pour garantir une morale élevée :

Même les pays barbares possèdent des religions pour discipliner les mœurs. Comment, avec sa civilisation de cinq mille ans, la Chine qui a été cultivée par d'innombrables sages, n'aurait-elle pas de religion/enseignement? À cause de la pauvreté et de la faiblesse du pays, mais aussi de la perte de la Voie durant la dynastie Qing, le peuple admire l'Europe et les États-Unis. Dans cette situation, s'il est bon d'œuvrer en faveur

15. Kang Youwei, «Kongjiaohui xu », p. 345-346. 


\section{Gan Chunsong}

d'un changement politique, comment est-il possible d'abandonner tout le processus de transformation par l'éducation hérité de plusieurs millénaires ${ }^{16}$ ?

Kang Youwei estimait que les Chinois de l'époque non seulement ne croyaient pas en un système gouverné par la loi, mais qu'ils manquaient également de conscience morale, ressemblant à un peuple non éduqué. Or, la construction d'un ordre social devait non seulement s'appuyer sur la loi, mais également sur la morale.

Pour que le peuple puisse respecter volontairement [les lois], il faut avoir une religion qui le gouverne de façon invisible. Ce n'est pas sans inconvénient. Pourtant, peu de gens préconisent la sagesse, tandis que beaucoup de gens médiocres se soucient de leur malheur et bonheur. Devant Dieu, on ne peut pas être infidèle. Le bien sera récompensé par le bien, le mal par le mal. Si tout cela pénètre le cœur du peuple, cela constituera alors le fondement des mœurs et le principe directeur des mentalités ${ }^{17}$.

C'est pourquoi Kang poursuivit son projet de fondation d'une religion confucéenne, recherchant une protection institutionnelle au sein du nouveau système politique et juridique et espérant, par la voie législative, ériger la religion confucéenne en religion d'État.

En 1913, lors du débat sur la Constitution au parlement national, Chen Huanzhang, Liang Qichao, Yan Fu et d'autres remirent au Sénat et à la Chambre des représentants une «Pétition en faveur d'une Église de la religion confucéenne». Rappelant que le confucianisme était autrefois la religion de l'État chinois, ils s'appuyaient sur «l'opinion du peuple», utilisant ainsi un procédé de la politique démocratique moderne, pour démontrer la position légale de la religion confucéenne:

Aujourd'hui, le régime d'État est la République, le peuple est le maître du pays. Il n'est pas permis d'agir contre la volonté du peuple [...]. L'esprit de la République repose sur la morale, or l'origine de la morale en Chine est la religion confucéenne, on ne saurait déterrer la racine ou boucher la source. Par conséquent, il est absolument nécessaire que la Chine institue la religion confucéenne comme religion d'État. Certains pensent que l'établissement d'une religion d'État serait contradictoire avec la liberté de croyance religieuse inscrite dans la Constitution provisoire. Ils ne se rendent pas compte que c'est faux. Dans notre pays, depuis l'Antiquité, la religion confucéenne est religion d'État, de même que depuis l'Antiquité, la liberté de croyance religieuse est accordée au peuple. À quel moment ces deux [principes] qui fonctionnent depuis plusieurs millénaires comme les articles d'une constitution non-écrite auraient-ils été incompatibles? Ce qui est aujourd'hui codifié dans la

16. Kang Youwei, «Zhonghua jiuguo lun», p. 325.

17. Ibid. 
Constitution n'est qu'un fait à l'œuvre depuis longtemps. La liberté de croyance religieuse est une politique passive tandis que l'instauration d'une religion d'État est une politique active. Les deux peuvent aller de pair sans inconvénient et se soutiennent mutuellement. [...] [C'est pourquoi il est demandé] d'inscrire dans la constitution la religion confucéenne comme religion d'État ${ }^{18}$.

Le lancement de cette pétition connut un véritable retentissement. Li Yuanhong (1864-1928), alors le vice-président de la République, suivi des gouverneurs et des préfets de plus de dix provinces (Zhejiang, Shandong, Hubei et Henan...), envoyèrent tous un télégramme pour apporter leur soutien. Tang Hualong (1874-1918), ministre de l'Éducation à cette époque, fut encore plus précis en déclarant:

Ces dernières années, le sujet qui suscite le plus de débat est celui du déclin moral, les jeunes agissant selon leur envie, sans règle à suivre. Les lettrés concernés n'en trouvent pas la cause. J'ai deux propositions pour résoudre ce problème: 1) dans les écoles primaires et secondaires, il faut apprendre l'ensemble des Classiques confucéens. De cette façon, les discours profonds et subtils des saints et des sages influenceront progressivement les jeunes et formeront ainsi leur caractère. C'est une proposition pour renforcer la racine ; 2) adopter le confucianisme comme religion d'État, en le mettant en œuvre par des rituels religieux, de sorte que les citoyens deviennent des fidèles religieux et observent les paroles et les conduites de Confucius comme des préceptes religieux. C'est une proposition pour établir la croyance ${ }^{19}$.

Cet extrait peut être perçu comme une critique à l'égard de l'action entreprise par Cai Yuanpei (1868-1940) visant à éliminer les pensées de Confucius du système d'éducation républicain ${ }^{20}$.

Si cette pétition fut accueillie positivement dans la société, de nombreuses voix, y compris religieuses, se firent également entendre contre elle. L'opposition du député He Wen était sans doute la plus aboutie:

1) la Chine n'est pas un pays religieux ; 2) Confucius n'est pas un maître religieux ; 3) la liberté de croyance religieuse est une prescription constitutionnelle, l'instauration

18. Zhongguo shehui kexueyuan jindaishi yanjiu suo, Kongjiaohui ziliao, p. 33-34.

19. Voir Ke Huang (ed.), Kongjiao shinian da shiji, volume Ge, p. 76.

20. En 1912, Cai Yuanpei, devenu ministre de l'Éducation, publia un À propos de l'orientation et des principes de nouvelle éducation, qui s'opposait à l'objectif final d'éducation établi en 1906. Il affirmait: «La loyauté envers l'empereur [était] incompatible avec le régime républicain, et la primauté accordée au confucianisme contraire à la liberté de croyance». Voir Cai Yuanpei, «Duiyu xinjiaoyu zhi yijian»,p. 136. En juillet 1912, la Conférence sur l'éducation qui s'ouvrit provisoirement vota un nouvel objectif d'éducation: «attacher de l'importance à l'éducation morale, compléter cette éducation par l'éducation scientifique, citoyenne et militaire, et la parfaire avec l'éducation esthétique». 


\section{Gan Chunsong}

de la religion confucéenne comme religion d'État serait incompatible avec la constitution; 4) la République se compose de cinq ethnies, et en dehors de la religion confucéenne, il existe toute sorte de religions telles le lamaïsme et l'islam: si la religion confucéenne était instituée comme religion d'État, cela risquerait de provoquer la déloyauté des Mongols et des Tibétains ${ }^{21}$.

Ces débats rejaillirent rapidement dans le travail de préparation de la constitution parmi les députés. Les deux parties campant sur leur position, d'aucuns proposèrent la formule «prendre la voie de Confucius comme grand fondement des mœurs ».

Le 13 octobre 1913, les propositions des parlementaires furent soumises au vote, en présence de quarante députés. La première proposition, « Il devrait être établi dans la constitution que la religion confucéenne est la religion de l'État», obtint huit votes. La seconde, «La République de Chine définit la religion confucéenne comme le grand fondement moral des relations humaines », reçut 15 voix ; 11 personnes se prononcèrent en faveur de la troisième proposition, «La République de Chine définit la religion confucéenne comme le grand fondement moral des relations humaines. Toutefois, du moment que les autres religions ne troublent pas l'ordre public, le peuple dispose de la liberté de croyance». Or, l'adoption d'une proposition parlementaire nécessitant une majorité des deux tiers, ces trois propositions furent toutes rejetées.

Le 28 octobre, après la deuxième lecture du «Projet de constitution de la République de Chine», Wang Rongbao (1878-1933) proposa de nouveau d'ajouter, à la suite de l'article 19, la formule: «L'éducation de la population prend la voie de Confucius comme grand fondement éthique». Le débat qu'il suscita aboutit à la révision suivante: «L'éducation de la population prend la voie de Confucius comme grand fondement pour la culture de soi (xiushen)». Trente et un députés ayant voté en sa faveur, la proposition fut adoptée ${ }^{22}$. Ce résultat était fort éloigné de ce qu'espéraient Kang Youwei et Chen Huanzhang.

La restauration monarchique de Yuan Shikai (1859-1916) entraîna un chaos politique durant les premières années de la République, la dissolution du nouveau parlement de la République en 1914 provoquant la suspension des travaux d'élaboration de la constitution. Après le décès de Yuan Shikai, l'ancien parlement national fut rétabli et la révision de la constitution reprit en 1916. Chen Huanzhang remit alors une nouvelle pétition, demandant aux deux Assemblées d'instaurer l'Église de la religion confucéenne comme religion d'État. Si les arguments avancés étaient dans l'ensemble similaires à ceux de la précédente pétition, Chen Huanzhang insistait particulièrement sur le lien unissant les

21. «Xianfa guiding guojiao wenti zhi shezhan», Shenbao, 3 octobre 1913.

22. Voir Huang Kewu, «Minguo chunian kongjiao wenti zhizheng lun». 
destinées de la religion confucéenne et de l'État, la religion confucéenne étant perçue comme le principe directeur pour préserver la «spécificité nationale» (guoxing).

Nous nous permettons de lancer un appel public aux compatriotes : pour éviter que la Chine ne soit conquise, il est nécessaire que la religion confucéenne devienne la religion de l'État; [...] Si nous, les citoyens, demandons d'instituer une religion d'État, ce n'est pas seulement par loyauté envers la religion confucéenne, mais encore plus sincèrement par loyauté envers la Chine. En effet, la religion confucéenne ne sera pas nécessairement ruinée si elle ne devient pas religion d'État; et une fois érigée en religion de l'État, elle ne sera pas en situation de monopole. [Avoir ou non le statut de religion d'État] ne causera pas de grand bénéfice ou de grand tort à la religion confucéenne. Mais pour notre peuple et notre pays, l'importance de cette affaire est sans comparaison. Si la religion confucéenne ne devient pas religion d'État, notre peuple ne sera plus le peuple chinois, notre pays ne sera plus la Chine mais un pays inférieur ${ }^{23}$.

Cette pétition remise au Parlement continuait de soutenir que la liberté de croyance et l'établissement d'une religion d'État n'étaient pas contradictoires. Les voix d'opposition étaient pourtant encore très fortes. Dans une pétition s'opposant à l'établissement de la religion confucéenne comme religion d'État, étaient ainsi avancés les effets néfastes qu'aurait une telle institutionnalisation : elle (1) provoquerait des querelles entre les religions ; (2) détruirait la concorde entre les cinq ethnies ; (3) enfreindrait la constitution provisoire de la République ; et (4) ferait obstacle à l'unité politique.

En résumé, la religion n'est pas un problème d'importance dans l'histoire de la Chine, il n'est pas indispensable d'instaurer une religion d'État. Mais une fois que la République est conçue comme composée de cinq ethnies, il est nécessaire de n'en pas instaurer. De plus, Confucius est un pédagogue et un maître en politique, non un maître religieux. Les chercheurs d'Orient comme d'Occident confirment tous ce point. Les savants et les lettrés de notre pays considèrent également Confucius comme un grand homme dans les domaines de l'éducation et de la politique, au premier rang dans toute l'histoire de notre pays. Comment peut-on imposer le nom de religion au confucianisme et jeter le trouble dans la compréhension de la réalité ? La demande d'instauration de la religion confucéenne comme religion d'État est issue d'une ignorance de la situation générale actuelle. Ses partisans ne comprennent ni ce qui fut Confucius, ni ce qui fait la religion ${ }^{24}$.

Pour Kang Youwei et Chen Huanzhang, le résultat de cette révision était encore plus éloigné de leur objectif.

23. Kongjiao hui ziliao.

24. Voir Shen Yunlong (ed.), Minguo jingshi wenbian, p. 5144. 


\section{Gan Chunsong}

Sans même évoquer l'instauration de la religion confucéenne comme religion d'État, l'article de compromis selon lequel «L'éducation de la population prend la voie de Confucius comme grand fondement pour la culture de soi» provoqua aussi des débats. Certains députés suggérèrent de le supprimer de la constitution, arguant que les idées de Confucius n'étaient adaptées ni au régime républicain ni au principe de la liberté de croyance; que la question de l'éducation de la population était administrative et ne devait pas être déterminée par la constitution; que la culture de soi relevait de la sphère morale et ne correspondait pas à l'essence de la constitution. Par ailleurs, l'éducation de la population étant obligatoire, si l'on inscrivait la voie de Confucius dans la constitution, alors les croyances de fidèles d'autres religions deviendraient illégales. Pour honorer Confucius, l'État pouvait recourir à d'autres voies, sans passer par cet article.

D’autres appelèrent à conserver ce qui avait été défini dans le projet initial de la constitution. Considérant que la voie de Confucius ne pouvait être perçue comme une religion, il n'y avait dès lors aucun rapport avec la liberté de croyance. L'ensemble du peuple continuait à croire en Confucius, la voie de Confucius constituant le fondement moral de la société. Supprimer cet article déjà inscrit dans la constitution initiale ne ferait que susciter des craintes, alors que d'autres constitutions à l'étranger présentaient ce genre de formule. Ils avancèrent même l'argument selon lequel certaines églises étrangères lisaient les Quatre Livres et les Cinq Classiques pour démontrer que la religion confucéenne ne provoquerait pas de différend avec les autres religions.

Les arguments des deux camps étaient approximativement semblables à ceux avancés lors de la première discussion. Aucune proposition n'obtenant la majorité aux deux tiers, des concessions furent faites pour parvenir au compromis suivant: d'une part, la suppression au sein du projet de la formule «L'éducation de la population prend la voie de Confucius comme grand fondement pour la culture de soi »; d'autre part, la modification de l'article 11, la «liberté de croyance religieuse» devenant «Le peuple de la République de Chine honore Confucius et dispose de la liberté de conviction religieuse, dans les limites fixées par la loi » ${ }^{25}$.

Loin d'être superficiels, les débats de 1913 et de 1916 sur l'inscription dans la constitution de la religion confucéenne comme religion d'État soulevaient de nombreuses questions, la première étant de déterminer si l'éthique confucéenne

25. Pour une description précise des débats sur l'établissement de la religion confucéenne comme religion d'État à travers les révisions successives de la constitution durant les premières années de la République, voir Yin Xiaohu, Jindai zhongguo xianzheng shi, p. 188-194. 
devait continuer à être le fondement de l'identité du pays. Selon les études menées par Jin Guantao et d'autres, la diffusion de l'idée de l'État moderne fit prendre conscience aux Chinois que «la nation pensée comme communauté morale pouvait être distinguée de l'éthique confucéenne ${ }^{26}$. C'est pourquoi l'incorporation de la religion confucéenne à l'identité de la nation n'était pas adaptée au régime républicain et ne correspondait pas non plus à l'état d'esprit des nouveaux intellectuels de l'époque. Pour Huang Jinxing, l'échec du projet de religion confucéenne est lié à deux forces principales: l'influence croissante du scientisme, mais aussi de nombreux événements politiques imprévus tels que la restauration monarchique de Yuan Shikai ${ }^{27}$.

Comme le montre l'analyse précédente, l'appel de Kang Youwei et de Chen Huanzhang en faveur d'une «religion d'État» n'avait pas pour visée concrète le monopole des rituels d'État par les intérêts confucianistes, mais cherchait à résoudre le problème entre la religion d'État et la liberté de croyance. Leur volonté de fonder une religion d'État n'était pas entièrement liée à des convictions religieuses, mais se fondait également sur l'identité de l'État-nation, tous deux espérant que la lecture des Classiques confucéens devienne un enseignement central du système d'éducation de la Chine moderne afin de préserver l'identité culturelle. Dans ce sens, les activités de Kang Youwei en faveur d'une religion confucéenne étaient davantage politiques que purement religieuses.

\section{Le confucianisme et «les études matérielles»}

Si l'horizon du confucianisme traditionnel résidait dans la figure du sage, son objectif réel était de promouvoir la figure de l' «homme de bien» (junzi). Cela explique que les talents sélectionnés par les examens impériaux n'étaient pas de simples gestionnaires spécialisés, mais - idéalement - des hommes possédant une compréhension profonde de la conception confucéenne de l'ordre.

Sous l'influence de cette conception, après la dynastie Han et l'instauration du système de sélection et de recommandation (chaju), puis après les dynasties Sui et Tang qui développèrent le système des examens impériaux, l'éducation en Chine, dans les écoles officielles ou dans les académies privées, devint une extension du système mandarinal. Cela favorisa une diffusion efficace des idées confucéennes, mais entraîna une uniformisation qui fit obstacle à la formation et la diffusion d'autres formes de connaissances.

Si des critiques furent de tout temps formulées à l'égard des examens impériaux, ce système n'avait pourtant jamais rencontré de réel défi. À partir

26. Jin Guantao et al., Guannianshi yanjiu, p. 243.

27. Huang Jinxing, Shengxian yu shengtu, p. 54. 


\section{Gan Chunsong}

de 1840, avec les défaites successives de la Chine, en particulier lors de la guerre sino-japonaise, les Chinois prirent conscience dans la douleur que le retard de la Chine était principalement lié à un décalage dans le domaine des savoirs, la Chine manquant de talents capables d'affronter les nouveaux défis. La réforme du système des examens impériaux et la refondation du système scolaire devinrent un consensus partagé par tous.

En apparence, la réforme du système d'éducation n'ébranlait pas directement le système de croyances chinois et parmi tous les plans de réforme proposés lors des Cent jours, les mesures visant à fonder des universités et des maisons d'édition officielles rencontrèrent le moins d'obstacles. La seule mesure qui fut véritablement mise en œuvre par le nouveau gouvernement à la fin des Qing fut d'ailleurs la suppression du système des examens impériaux en 1905. Kang Youwei, Zhang Zhidong et d'autres voyaient avant tout dans la fondation de nouvelles écoles des institutions complémentaires: en associant aux valeurs confucéennes les techniques scientifiques occidentales, la Chine ne devait plus se laisser distancer. Ils n'imaginaient pas que la réforme éducative constituait le premier pas vers le déclin du confucianisme.

Dans l'esprit de Kang Youwei, l'enjeu de la religion confucéenne était de faire la synthèse entre «transformation par l'éducation » (jiaohua) et «religion» (zongjiao), tandis que les examens impériaux devaient amener à la séparation entre savoirs et croyances. L'éducation n'était donc plus seulement la mise en œuvre concrète de la «transformation par l'éducation», mais bien un élément essentiel dans l'apprentissage de connaissances. En 1898, dans une requête célèbre, Kang affirme que la sélection des lettrés en fonction de l'essai en huit parties éloigne des anciens textes sacrés et empêche de comprendre le monde environnant au point d'avoir occasionné les défaites militaires de la Chine. D'où la nécessité de sa suppression:

Désormais, à l'intérieur du pays, on apprendra la culture chinoise, on étudiera les idées classiques, l'histoire du pays, les anecdotes ainsi que la nomination et la classification des choses, afin de devenir une personne aux talents utiles. Vers l'extérieur, on recourra aux sciences des autres pays, on étudiera la technologie, la physique, la politique-éducation et le droit, afin d'avoir une connaissance globale ${ }^{28}$.

Liang Qichao souligna lui aussi le contraste entre les principes des examens impériaux fondés sur l'idée que «l'homme de bien n'est pas un ustensile destiné à un seul usage » (junzi bu qi, la vertu morale, prévaut sur les talents de l'expert) ${ }^{29}$ et la technicité de l'éducation occidentale. Il avait par ailleurs conscience du

28. Kang Youwei, «Qing fei bagu shitie kaifa shishi gaiyong celun zhe», p. 79-80.

29. Citation du Lunyu, II, 12 (traduction Anne Cheng, p. 35). 
lien unissant les examens impériaux et la diffusion du confucianisme, y compris dans la décadence: «Cet enseignement grandiose recourt pour se perpétuer au système des examens mandarinaux, qui est lui-même en déclin. Ce qui dépend du système des examens mandarinaux est proche de la mort ${ }^{30}$. Liang Qichao s'inquiétait également de l'usage fort répandu à l'époque qui consistait à reprendre les paroles des sages sans que cela permette de résoudre les problèmes de l'époque. Pour Liang, ces tendances condamnaient le confucianisme à court terme. La difficulté était dès lors de savoir comment concilier le développement d'un enseignement nouveau tout en préservant l'esprit du confucianisme.

Concernant la mise en œuvre de la réforme de l'éducation, l'argument initial de Kang Youwei était de former des talents adaptés à leur époque, connaisseurs autant de la Chine que de l'Occident. En juin 1898, dans le mémoire rédigé au nom de Song Bolü, il écrit:

Le manque de talents en Chine est dû au fait que les savoirs chinois et occidentaux ne peuvent communiquer. Ceux qui sont éduqués dans le système des examens mandarinaux ne connaissent rien du savoir occidental, tandis que ceux issus des écoles modernes ne comprennent rien du savoir chinois. Le savoir chinois est la substance, le savoir occidental est le fonctionnement. Sans substance, on ne peut pas s'établir, sans fonctionnement on ne peut pas pratiquer ${ }^{31}$.

Pour résoudre ce problème, le point essentiel était de réformer les modes d'examen. L'idée de Kang était de combiner l'examen traditionnel et un nouvel examen portant sur l'économie et la politique, de «faire passer à tous les candidats une épreuve pratique (celun)». Il s'agissait «d'évaluer la connaissance des idées classiques et de l'histoire» et parallèlement d' «évaluer la connaissance des affaires politiques actuelles ainsi que les savoirs spécifiques » : on formerait ainsi des personnes au «savoir global».

Concrètement, Kang appelait à imiter l'éducation obligatoire déjà promue en Occident tout en tenant compte de la spécificité chinoise et en proposant de transformer les temples populaires en écoles. Ainsi, dans un mémoire au trône de juillet 1898, il affirmait:

Les Chinois ordinaires se laissent souvent tromper par les croyances envers les esprits et génies, les temples dédiés à des cultes immoraux sont partout dans le pays. Par exemple, dans le Guangdong, mon pays natal, chaque canton a plusieurs temples et chaque temple possède des propriétés communes. On devrait transformer les temples en écoles et rendre les propriétés communes à l'usage public. On devrait aussi suivre le modèle de l'antiquité chinoise et celui des pays occidentaux qui oblige

30. Liang Qichao, «Xuexiao zonglun», p. 19.

31. Kang Youwei, Kang Youwei quanji, tome 4, p. 306. 


\section{Gan Chunsong}

les enfants de six ans à aller à l'école primaire et leur enseigne les mathématiques, la technologie, la langue et la littérature. Les parents des enfants qui ne se rendraient pas à l'école seraient punis ${ }^{32}$.

En réalité, si Kang Youwei avait conscience des relations d'interdépendance entre le confucianisme, le bouddhisme, le taoïsme et les cultes populaires locaux dans le système religieux traditionnel en Chine, il avait une vision négative des croyances populaires, ne s'apercevant pas du rôle de ces dernières dans la diffusion des conceptions confucéennes.

En appelant au dialogue entre la Chine et l'Occident, Kang Youwei accordait en fait un espace d'existence aux écoles de type occidental : c'était là le modèle qui se profilait derrière le modèle japonais. Kang accepta ainsi d'occuper le poste de directeur pédagogique à l'Université impériale de Pékin (Jingshi daxuetang), afin de piloter directement le développement d'un enseignement de type nouveau en Chine et d'offrir une assise institutionnelle à ses propositions. Au départ, Kang et Liang furent chargés d'élaborer les règlements de cette université. Mais des problèmes liés à l'élaboration des manuels et aux limites du pouvoir du directeur pédagogique provoquèrent la mise à l'écart de Kang, son objectif ne pouvant ainsi être réalisé.

Si Kang Youwei attribua cet échec à des rivalités politiques, la raison principale résidait dans l'attitude adoptée à l'égard de la science occidentale et de l'étude des Classiques confucéens. Le camp des opposants à Kang ne souhaitait pas voir l'école moderne des Classiques confucéens (jinwen jingxue), défendue par ce dernier, placée à la tête des études confucéennes; ses adversaires considéraient par ailleurs que la réforme de Kang était trop radicale et occidentalisée ${ }^{33}$. Prise dans ce débat, la construction de l'université ne connut pas de réel développement. Or, la suppression des examens impériaux devenant à l'ordre du jour, l'enjeu ne fut plus celui des disputes internes au système confucéen, mais bien la survie des études confucéennes au sein du nouveau système d'éducation.

Dans ce contexte, Zhang Zhidong et d'autres considéraient que remplacer l'essai en huit parties par une dissertation sur la stratégie et une épreuve sur l'économie ne modifierait absolument pas la dépendance à l'égard des examens impériaux. Il fallait par ailleurs résoudre le problème des débouchés des étudiants des écoles de type nouveau. C'est pour cette raison que Zhang Zhidong, Yuan Shikai et d'autres considérèrent que tant que les examens impériaux existeraient, les écoles ne pourraient être acceptées. La promotion du système des écoles passait nécessairement par l'abolition des examens impériaux. Dans le mémoire

32. Kang Youwei, ibid., tome 4, p. 318.

33. Mao Haijian, «Jingshi daxuetang de chujian», p. 300-301. 
qu'ils rédigèrent ensemble en 1903, intitulé «Requête au trône en faveur de la suppression progressive des examens impériaux », ils devinrent des réformateurs radicaux :

Une bonne gouvernance exige des gens compétents. Ceux-ci sont formés à l'école, dans le passé comme dans le présent, en Chine comme à l'étranger. Si le système des examens mandarinaux n'est pas abandonné, le système moderne de l'école ne pourra se développer. Les étudiants n'auront jamais de savoirs concrets, le pays ne possèdera jamais de personnes compétentes pour répondre aux besoins du temps présent. La Chine $[\ldots]$ ne pourra jamais concurrencer les autres pays ${ }^{34}$.

Mais quelle place accorder aux Classiques confucéens dans les écoles de type nouveau ? Zhang proposait de créer un cours d'étude des Classiques confucéens doté d'une place spécifique dans les universités, car de ce cours dépendrait en partie la survie des croyances des Chinois:

Pendant leur formation à l'école, il est absolument nécessaire d'enseigner aux élèves et de leur faire lire les Classiques. La quantité de ces Classiques à lire pourra varier selon les différentes écoles, de même que la profondeur de l'enseignement. Il n'est pas utile de les standardiser. Même pour ceux qui travaillent après l'école primaire, il leur est nécessaire de lire les passages importants des Classiques et d'entendre les idées essentielles de l'enseignement saint, afin qu'ils puissent établir leur nature fondamentale ${ }^{35}$.

Malgré son exil à l'étranger après la réforme des Cent jours, Kang Youwei, fin connaisseur des caractéristiques de la religion en Occident, poursuivit sa réflexion sur la voie de développement propre à la Chine. Prêtant attention à la différence entre éducation (jiaoyu) et transformation par l'éducation (jiaohua), il proposa non seulement de différencier le sens de ces deux mots mais également de les séparer dans la conception du système d'administration.

Dans «Guanzhi yi» (Discussion sur le système des fonctionnaires), Kang Youwei distingue clairement l'enseignement de la religion. Dans son projet, le ministère de la Culture aurait en charge les activités concernant les écoles et l'art d'enseigner, c'est-à-dire «toutes les affaires concernant les écoles publiques et leurs enseignants, leurs bibliothèques, bâtiments, assemblées et musées ainsi que les bourses aux étudiants ", tandis que le ministère de la Religion s'occuperait principalement des croyances et de la transformation par l'éducation (jiaohua):

Comme le montrent les constitutions d'autres pays, si la croyance religieuse est le choix autonome de chacun, chaque pays possède une religion d'État, appropriée à ses

34. Zhang Zhidong \& Yuanshikai, «Zouqing dijian keju zhe».

35. Zou ding xuetang zhangcheng - xuewu gangyao, Hubei xuewuchu, 1903. 


\section{Gan Chunsong}

politiques, à la mentalité du peuple et à ses coutumes, qui ne peut être abandonnée. Pour cette raison, tous les pays ont un ministère de la Religion afin de réguler les affaires religieuses. En Chine, la politique, les normes, le système scolaire ainsi que la sélection des fonctionnaires relèvent tous du confucianisme, le ministère des Rites actuel est en réalité le ministère de la Religion ${ }^{36}$.

Dans ce projet, le ministère de la Religion devait se charger des affaires concernant la religion et de l'organisation des activités d'éducation de la société (jiaohua), mais plus de l'enseignement dans les écoles. Cette stratégie de réforme de l'éducation présentait, comme on le voit, une cohérence interne avec le projet qu'avait Kang de fonder une Église de la religion confucéenne.

Parce que Kang essayait d'embrasser en un seul mouvement tous les problèmes rencontrés par le confucianisme, il fut perçu par certains comme un opposant farouche au système politique moderne et aux connaissances scientifiques. Cette vision négligeait ses efforts pour intégrer la science moderne aux études confucéennes, sa vision de la science et même de l'économie présentant une grande originalité.

Dans son Wuzhi jiuguo lun (Sur le salut matériel du pays) rédigé autour de 1904 et publié en 1908, Kang Youwei estimait que la force d'un pays ne dépendait pas principalement de sa formation morale, mais de la production matérielle et de la «science matérielle» qui en était le fondement. Il considérait que depuis le mouvement de la Renaissance en Europe, la culture et la pensée avaient connu trois grandes mutations : «un savoir sur les humains au début, puis pendant plusieurs décennies la connaissance des nations et des citoyens, enfin l'étude des conditions matérielles $\gg{ }^{37}$. Avant la fondation de la République, le souci principal de Kang était la prégnance des examens impériaux et le faible intérêt pour les connaissances scientifiques modernes. Mais après la fondation de la République, devant les mises en cause de la religion de Confucius émanant notamment de Cai Yuanpei, Kang revint à la question de la lecture des Classiques confucéens dans les écoles nouvelles.

Suite à la suppression par le ministère de l'Éducation de l'étude des Classiques confucéens dans les écoles primaires et secondaires en 1912, Kang Youwei adressa à de nombreuses reprises des lettres aux principaux initiateurs de cette mesure, dont Yuan Shikai, pour exprimer fortement son opposition. En 1916, après la nomination de Fan Yuanlian (1875-1927) comme ministre de l'Éducation et sa décision de transformer le cours de lecture des Classiques confucéens en un cours de culture de soi, Kang Youwei écrivit de nouveau une

36. Kang Youwei, «Guanzhi yi», p. 310 .

37. Kang Youwei, «Wuzhi jiuguo lun», p. 72. 
lettre où il estimait indissociables le perfectionnement de soi et la lecture des Classiques.

Face à l'argument qui pointait l'absence de Classiques dans les écoles étrangères, Kang répondait: l'institution de l'église en Occident avait déjà résolu le problème de la lecture des Classiques; or la Chine ne disposait ni d'institution équivalente à l'église en Occident, ni du système de lecture dominicale de la Bible.

[En interdisant la lecture des Classiques au sein des écoles], la jeune génération ne connaîtra jamais les Classiques confucéens. Ces derniers seront amenés à disparaittre dans vingt ou trente ans. Il s'agira là d'une méthode efficace et rapide pour éteindre la religion confucéenne. Si cela se produisait, à quoi se rattacheraient les mentalités et les mœurs des gens de tout le pays? Le recours au bouddhisme exige la sortie de la famille et la vie extra-mondaine, ce qui est difficilement acceptable pour les gens. Le recours au christianisme interdit de faire un culte devant les tombeaux [des ancêtres], ce qui ne convainc pas non plus. Les gens vont-ils alors suivre les animaux sans religion $?^{38}$

Pour Kang, l'apprentissage des Classiques par les enfants ne nécessitait pas de prêter trop attention au fait de savoir s'ils comprenaient ces textes, car leur sens se manifesterait naturellement lors de leur arrivée à l'âge adulte. Cet apprentissage conduirait nécessairement les Chinois à construire les croyances morales élémentaires. Kang savait qu'en Occident, la lecture de la Bible était essentiellement une affaire qui concernait l'Église et ne faisait pas partie du contenu enseigné à l'école. Mais en raison de l'échec de l'instauration de la religion confucéenne comme religion d'État, l'enseignement des Classiques à l'école devint une position à laquelle il se tint fermement. Kang s'enferma donc dans une contradiction à laquelle il ne pouvait échapper : il commença par espérer une différenciation entre les croyances et les connaissances, mais le domaine des croyances ne pouvant être établi, il fut contraint d'essayer d'intégrer les croyances à l'intérieur du système de valeurs moderne.

Si Kang était insatisfait de la disparition des Classiques dans l'enseignement scolaire, il critiquait aussi le fait que l'éducation républicaine ne fût pas parvenue à enseigner les bons aspects de l'Occident:

Les points forts de l'Europe et des États-Unis résident d'abord dans le développement matériel [...], ensuite dans une éducation obligatoire qui s'applique à tous les citoyens et augmente de manière générale leur niveau de connaissance. Or, en Chine, on parle tous les jours de développer l'éducation, mais on ne réalise jamais ces deux points, c'est pourquoi on en voit les inconvénients plutôt que les avantages. Si l'on

38. Kang Youwei, «Zhi jiaoyu zongchang yuan jingsheng shu», p. 322. 


\section{Gan Chunsong}

veut vraiment promouvoir l'éducation, il suffit de demander aux autorités locales d'ouvrir le plus possible d'écoles pour les études matérielles, et de rendre l'éducation obligatoire dans tous les districts, cantons et villes. Quelques années plus tard, les études matérielles se seront progressivement développées et les enfants auront tous des connaissances générales ${ }^{39}$.

En novembre 1923, lors d'un discours prononcé à Xi'an, Kang insistait encore sur sa position:

Notre pays est fort en études spirituelles, mais manque d'études matérielles. Sans recherche scientifique ni étude matérielle, il est pauvre et faible et ne peut devenir prospère et puissant. Aujourd'hui, il faut profiter du développement matériel de l'Europe et des États-Unis, promouvoir la science, afin de corriger les défauts de notre pays. Quant à la transformation morale par l'éducation, elle nous appartient de manière intrinsèque, il faut faire de notre mieux pour la défendre et ne jamais l'abandonner. [...] À l'extérieur, nous devons poursuivre les sciences de l'Europe et des État-Unis, tout en défendant, à l'intérieur, la religion confucéenne en tant que quintessence nationale ${ }^{40}$.

Cependant, la réalité ne suivit pas la direction envisagée par Kang. Pour empêcher que le nouveau système d'éducation prive le confucianisme de soutien, l'Église de la religion confucéenne décida en 1923 de fonder une université d'enseignement de Confucius. Comme nous le montre l'annonce de recrutement parue dans le numéro 5 du deuxième tome du Jingshi bao, les sections universitaires au sein de cet établissement comportaient un département d'études confucéennes, un département de littérature ainsi qu'un département de droit. En septembre 1923, l'université fit sa rentrée, et dans l'annonce qu'elle fit paraître après sa fondation, elle dénonçait violemment le fait que l'éducation moderne utilisât les ressources de l'État pour détruire l'enseignement de Confucius. Toutefois, des documents précis concernant cette université restent à découvrir.

En intégrant de nouvelles connaissances scientifiques, le nouveau système d'éducation n'eut de cesse d'éroder le caractère sacré et l'espace d'existence des études confucéennes, les écoles modernes devenant finalement le lieu de déconstruction de la signification du confucianisme. La synthèse effectuée par Cai Yuanpei lors des débuts de l'Université de Pékin témoigne tout à fait de cette tendance. Cai évoquait ainsi «trois étapes»:

Depuis sa fondation jusqu'à la première année de la République, durant une dizaine d'années, [...] le principe de notre école était «le savoir chinois comme substance, le savoir occidental comme fonctionnement». Les enseignants et les apprenants

39. Kang Youwei, «Luan hou zui yan», p. 156.

40. Kang Youwei, «Chang'an jiangyan lu», p. 278. 
mettaient tous l'accent sur les études anciennes; le savoir occidental [...] apparaissait comme une décoration [...] De la première année à la sixième année de la République, [...] émergea un élan visant à abandonner complètement les études anciennes, [...] à ce moment, le savoir chinois recula et se retrouva en situation de décoration. [...] Depuis la sixième année de la République, on cherche plutôt à faire communiquer savoir chinois et savoir occidental. Il est vrai que la science inventée par l'Occident exige la méthode occidentale pour faire les expériences, mais les matières chinoises, c'est-à-dire les études propres à la Chine, doivent aussi être soumises au traitement par la méthode scientifique ${ }^{41}$.

\section{Conclusion}

Dans la Chine traditionnelle, le confucianisme représente la synthèse entre des croyances, des connaissances et une morale. La religion et la science occidentales pénétrèrent conjointement en Chine, $\mathrm{y}$ apportant un nouveau mode de pensée né du mouvement des Lumières, qui sépare les croyances religieuses de la science empirique. Kang Youwei fut précurseur lorsqu'il tenta d'utiliser la différenciation occidentale entre la religion et les savoirs pour reconstruire un avenir au confucianisme. Il souhaitait ainsi que le confucianisme devînt une religion, se proclamant le Martin Luther de la religion confucéenne. Bien qu'il analysât de manière approfondie la différence entre la religion confucéenne (un enseignement de la voie humaine) et les autres religions (un enseignement de la voie divine), cela ne l'empêcha pas d'importer les églises, les préceptes religieux et même les actions de prosélytisme communes aux religions pour fonder son Église de la religion confucéenne. Il s'efforça également de promouvoir activement l'instauration d'une religion d'État dans le système de gouvernement constitutionnel de la République.

Cependant, ayant pour objectif la formation morale, le modèle d'éducation confucéen se distinguait du système d'éducation moderne dont le cœur était la formation professionnelle et la recherche scientifique. Il était donc perçu comme une raison essentielle dans le manque de compétitivité internationale de la Chine. Kang Youwei s'efforça de concilier éducation confucéenne et système d'enseignement moderne, mais après la fondation des nouvelles écoles, le confucianisme fut transformé en un système de connaissances et démembré entre les différentes disciplines modernes, en particulier après la suppression de l'étude des Classiques confucéens. La légitimité des valeurs confucéennes ne put par conséquent être préservée.

Comme le révèle le projet de réforme de Kang Youwei, le confucianisme tel qu'il le concevait devait différencier croyances et connaissances. Toutefois,

41. Cai Yuanpei, «Beida chengli ershiwu zhounian jinianhui kaihuici», p. 295-296. 
sa conception présentait une profonde contradiction: dans une époque gagnée de jour en jour par un élan rationaliste, Kang souhaitait fonder une religion confucéenne selon le modèle des églises en Occident, agissant ainsi dans le sens inverse de l'histoire. Par ailleurs, ses projets et actions demeuraient liés au soutien du pouvoir, à la dépendance à l'égard de l'empereur Guangxu pendant la réforme des Cent jours, aux tentatives d'instaurer une religion d'État sous la République. Cette caractéristique ne s'accordait pas non plus avec la tendance moderne à la séparation entre le politique et le religieux. La conséquence directe fut que les nouvelles classes d'intellectuels firent du confucianisme l'opposé de la démocratie et de la science. C'est pourquoi la réforme de Kang Youwei, loin d'aider le confucianisme à sortir de son impasse, contribua à l'inverse à ce que celui-ci perde son mode d'existence propre et ne puisse s'adapter à la vague de la modernité.

(Traduit du chinois par Aurore Merle et Guillaume Dutournier) 
Kang Youwei et le destin moderne du confucianisme

\section{BibliographIE}

Cheng Anne, Histoire de la pensée chinoise, Paris, Seuil, 1997.

CAI Yuanpei 蔡元培, «Duiyu xinjiaoyu zhi yijian» 對於新教育之意見, Cai Yuanpei quanji 蔡元培全集, éd. par Gao Pingshu 高平叔, Pékin, Zhonghua shuju, 1984, vol. 2, p. 130-137.

- 一, «Beida chengli ershiwu zhounian jinianhui kaihuici»北大成立二十五周年 紀念會開會詞, Cai Yuanpei quanji 蔡元培全集, éd. par Gao Pingshu 高平叔, Pékin, Zhonghua shuju, 1984, vol. 4, p. 295-297.

ChEN Xiyuan 陳熙遠, “"Zongjiao”: yige Zhongguo jindai wenhuashi shang de guanjianci», “宗教”- - 一個中國近代文化史上的關鍵詞, Xin shixue 新史學 (13) 4, 2002, p. 37-66.

Confucius, Les Entretiens de Confucius, trad. par Anne CHEng, Paris, Seuil, 1981.

Ding Wenjiang 丁文江 \& ZHAO Fengtian 趙豐田 (éds.), Liang Qichao nianpu changbian 梁啟超年譜長編, Shanghai, Shanghai renmin chubanshe, 1983.

Guangxu zhengyao 光緒政要, tome 29, Shanghai, Shanghai chongyitang, 1909.

HuANG Jinxing 黃進興, Shengxian yu shengtu 聖賢與聖徒, Taipei, Yunchen wenhua shiye youxian gongsi, 2001.

Huang Kewu 黃克武, «Minguo chunian kongjiao wenti zhizheng lun (1911-1917)»民 國初年孔教問題之爭論 (1911-1917), Guoli taiwan shifan daxue lishi xuebao 國 立臺灣師範大學歷史學報 12,1984,p. 197-223.

JIN Guantao 金觀濤 et al., Guannianshi yanjiu : Zhongguo xiandai zhongyao zhengzhi shuyu de xingcheng 觀念史研究: 中國現代重要政治術語的形成, Pékin, Falü chubanshe, 2009.

KANG Youwei 康有為, Kang Youwei quanji 康有為全集, Pékin, Zhongguo renmin daxue chubanshe, 2007, 12 tomes.

- - 《 Jiaoxue tongyi» 教學通義, in Kang Youwei quanji 康有為全集, Pékin, Zhongguo renmin chubanshe, 2007, tome 1, p. 17-58.

- - , 《Shili gongfa quanshu» 實理公法全書, in Kang Youwei quanji 康有為全集, Pékin, Zhongguo renmin chubanshe, 2007, tome 1, p. 145-160.

- - « Yu Zhu Yixin lun xue shu du» 與朱一新論學書牘, in Kang Youwei quanji 康 有為全集, Pékin, Zhongguo renmin chubanshe, 2007, tome 1, p. 314-335.

- - 《 Qing fei bagu shitie kaifa shishi gaiyong celun zhe» 請廢八股試貼楷法試 士改用策論折in Kang Youwei quanji 康有為全集, Pékin, Zhongguo renmin chubanshe, 2007, tome 4, p. 78-80.

- - « Guanzhi yi» 官制議, in Kang Youwei quanji 康有為全集, Pékin, Zhongguo renmin chubanshe, 2007, tome 7, p. 229-341.

- - «Wuzhi jiuguo lun» 物質救國論, in Kang Youwei quanji 康有為全集, Pékin, Zhongguo renmin chubanshe, 2007, tome 8, p. 61-101.

- - «Zhonghua jiuguo lun»中華救國論, in Kang Youwei quanji 康有為全集, Pékin, Zhongguo renmin chubanshe, 2007, tome 9, p. 309-329.

- - , «Yu Chen Huanzhang shu» 與陳煥章書, in Kang Youwei quanji 康有為全集, Pékin, Zhongguo renmin chubanshe, 2007, tome 9, p. 337-338. 


\section{Gan Chunsong}

- - « Kongjiaohui xu» 孔教會序, in Kang Youwei quanji康有為全集, Pékin, Zhongguo renmin chubanshe, 2007, tome 9, p. 343-346.

- - , «Luan hou zui yan» 亂後罪言, in Kang Youwei quanji 康有為全集, Pékin, Zhongguo renmin chubanshe, 2007, tome 10, p. 154-157.

- - , «Zhi jiaoyu zongchang yuan jingsheng shu» 致教育總長範靜生書, Kang Youwei quanji 康有為全集, Pékin, Zhongguo renmin chubanshe, 2007, tome 10, p. 321-325.

- - « Chang'an jiangyan lu» 長安講演錄, in Kang Youwei quanji 康有為全集, Pékin, Zhongguo renmin chubanshe, 2007, tome 11, p. 271-209.

Ke Huang 柯璜 (éd.), Kongjiao shinian da shiji 孔教十年大事記, volume Ge 革, 1924.

LiANG Qichao 梁啟超, «Xuexiao zonglun» 學校總論, in Liang Qichao 梁啟超, Liang Qichao quanji 梁啟超全集, éd. par ZHANG Pinxing 張品興, Pékin, Beijing chuban, 1999, vol. 1, p. 17-21.

- - «Baojiao fei suoyi zun Kong lun» 保教非所以尊孔論, in Liang Qichao 梁啟超, Liang Qichao quanji 梁啟超全集, éd. par ZHANG Pinxing 張品興, Pékin, Beijing chubanshe, 1999, vol. 3, p. 765-770.

MAO Haijian 茅海建, «Jingshi daxuetang de chujian - lun Kang Youwei pai yu Sun Jia'nai pai zhi zheng» 京師大學堂的初建——論康有為派與孫家舅派之爭, Beida shixue北大史學 13, 2008, Pékin, Beijing daxue chubanshe, p. 234-302.

PeNG Guangyu 彭光譽, Shuo jiao 說教, Beijing, Zongli geguo shiwu yamen, 1896.

SHEN Yunlong 沈雲龍 (ed.), Minguo jingshi wenbian (jiaotong, zongjiao, daode) 民國 經世文編(交通、宗教、道德), Taipei, Wenhai chubanshe, 1970.

《Xianfa guiding guojiao wenti zhi shezhan» 憲法規定國教問題之舌戰, Shenbao 申 報, 3 octobre 1913 .

YIN Xiaohu 殷嘯虎, Jindai zhongguo xianzheng shi 近代中國憲政史, Shanghai, Shanghai renmin chubanshe, 1997.

ZHANG Zhidong 張之洞 \& YUAN Shikai 袁世凱, «Zouqing dijian keju zhe» 奏請遞減 科舉折, Guanxu zhengyao 光緒政要, vol. 29, Shanghai, Congyitang, 1909.

Zhongguo shehui kexueyuan jindaishi yanjiu suo 中國社會科學院近代史研究所, Kongjiaohui ziliao 孔教會資料, Pékin, Zhonghua shuju, 1974.

Zou ding xuetang zhangcheng - xuewu gangyao 奏定學堂章程一一學務綱要, Hubei xuewuchu 湖北學務處, 1903 . 
Kang Youwei et le destin moderne du confucianisme

\section{GlossaIRE}

bagu 八股

Cai Yuanpei 蔡元培

celun 策論

chaju 察舉

Chen Huanzhang 陳煥章

Chunqiu 春秋

daoxueke 道學科

datong 大同

dijiao 帝教

Fan Yuanlian 範源濂

gaizhi 改制

Guangxu 光緒

guoxing 國性

guwen jingxue 古文經學

He Wen 何雯

housheng 後聖

Huang Jinxing 黃進興

Huang Zunxian 黃遵憲

jiao 教

jiaohua 教化

jiaoyi 教義

jiaoyu 教育

jiaozhu 教主

Jingshi bao經世報

Jingshi daxuetang 京師大學堂

jinwen jingxue 今文經學

junzi 君子

junzi bu qi 君子不器

Kang Youwei 康有為

keju 科舉

kongjiao 孔教

kongjiaohui 孔教會

Li Yuanhong 黎元洪

Liang Qichao 梁啟超

Liji 禮記

lijiao 禮教

Liyun 禮運

Lunyu 論語

Peng Guangyu 彭光譽

rendao 人道

rujiao 儒教

Shang qingdi di er shu 上清帝第二書 


\section{Gan Chunsong}

Shang qingdi di san shu 上清帝第三書 shengping昇平

shenjiao 神教

shijiao 師教

sishu 四書

taiping 太平

Tang Hualong 湯化龍

Wang Rongbao 汪榮寶

$\mathrm{wu}$ 巫

xiushen 修身

Yan $\mathrm{Fu}$ 嚴復

Yansheng Gong 衍聖公

Yuan Shikai 袁世凱

Zhang Daoling 張道陵

Zhang Zhidong 張之洞

zheng 政

Zhongyong 中庸

Zhu Yixin 朱一新

zong 宗

zongjiao 宗教 